\section{Strategies to combat medical misinformation on social media}

\author{
Samuel P Trethewey
}

The proliferation of medical misinformation on social media is a growing, global public health concern. ${ }^{1}$ The medical community is responding to this pervasive threat; earlier this year, the Chief Executive of the American Medical Association wrote to the leading technology companies calling for more action to 'ensure that users have access to scientifically valid information on vaccinations'. ' Similarly, the editors of leading cardiology journals recently collaborated to 'sound the alarm' on this issue and call for a coordinated effort from purveyors of web-based media to help to address medical misinformation. ${ }^{3}$ It is likely that a collaborative, interdisciplinary approach, using a wide range of interventions, will be required to counter the spread of medical misinformation on social media. ${ }^{4}$ This editorial discusses potential strategies to help to address this important public health issue.

\section{CAREFUL DISSEMINATION}

Careful dissemination of medical research is paramount in the fight against medical misinformation. Evidence suggests that research findings are often exaggerated or misrepresented in press releases and news media, ${ }^{5}$ and one of the main factors associated with 'spin' in press releases is 'spin' in article abstract conclusions. ${ }^{6}$ It is crucial that research findings are presented in an accurate, unbiased and balanced way in the biomedical literature, if we are to expect news media journalists and the general public to interpret these findings appropriately. A recent randomised trial demonstrated that small changes in press release headlines, in addition to explicit caveats/causality statements in the main text of press releases, can improve the accuracy of subsequent news headlines and stories. ${ }^{7}$ A report published in 2017 by the Academy of Medical Sciences contains detailed recommendations for researchers, press offices and journalists to guide accurate communication of scientific information to the public. ${ }^{8}$ Moreover, using evidence-based frameworks, such as the Disseminating Research Information through Facebook and Twitter (DRIFT)

Correspondence to Dr Samuel P Trethewey, Oak Tree Surgery, Liskeard, Cornwall, UK;

s-trethewey@doctors.org.uk framework, may help to standardise the way in which research findings are communicated to the general public via social media. ${ }^{9}$

Medical journals may be able to help to address medical misinformation. ${ }^{10}$ Many journals are already actively engaged in education and research dissemination via social media; journals could further engage directly with the public by more frequently curating accurate and accessible lay summaries of important research findings. In addition, journal editors could commission articles reviewing specific controversial health topics, as well as inviting submissions focussing on interventions to combat medical misinformation online. ${ }^{10}$

\section{EXPERT FACT-CHECKING}

A possible countermeasure is to perform targeted, expert fact-checking of social media posts. The method by which expert fact-checking is performed can mirror the open peer-review process used by medical journals: independent experts can be identified and asked to publicly review the content of social media posts to determine their accuracy. Specific fact-checking processes can be tailored to individual social media platforms. For example, labelling tweets with a 'Reviewed Content' status, following a formal peer-review process, could be a way to flag content as 'fact-checked' on Twitter. ${ }^{11}$ Experts could critically appraise a tweet and produce a written response comprising a brief, lay summary of the evidence in addition to a detailed, referenced, evidence review. This review could be directly linked to the original tweet, to provide users with quick access to fact-checked information. A key consideration is how to best identify content for peer-review: algorithm-based approaches, followed by robust screening processes, may help to identify popular tweets containing specific health-related content of public health importance.

There is an urgent need for more research to identify the most effective methods for expert fact-checking of health claims on social media. Current evidence suggests that to maximise the impact of the fact-checking process, corrective information should come from credible expert sources and specific health messages should be simple and should 'focus on the facts to be communicated rather than the misinformation to be corrected', to minimise the 'backfire effect' in which corrections paradoxically strengthen misperceptions. ${ }^{12} 13$ However, recent findings suggest that even simple 'false-tag' retractions (messages repeating a false claim while tagging it as false) can reduce belief in false claims, without any evidence of the 'backfire effect', while short-format (140 characters) refutations maybe even more effective. ${ }^{14}$

\section{SOCIAL MEDIA CAMPAIGNS}

Research is urgently needed to identify effective ways to infiltrate 'information silos' and 'echo chambers' to counter selective exposure, minimise confirmation bias and encourage balanced debate on topics of public health importance. One way to reach specific audiences may be to collaborate with social media influencers or key opinion leaders. For example, collaborating with 'mommy bloggers' on specific health promotion campaigns may help to facilitate dissemination of accurate, evidence-based health information to target audiences. ${ }^{15}$ Burke-Garcia et $a l^{15}$ suggest that such collaborations 'can leverage the established, powerful, wellused and trusting online social networks established on mommy blogs'. Similarly, expert health organisations could consider forging alliances with social media influencers and key opinion leaders, to run targeted health promotion campaigns. Lutkenhaus et $a l^{16}$ recently demonstrated, using network and content analysis of tweets related to vaccine hesitancy in the Netherlands, that it is possible to identify online communities with specific health perceptions and identify social media influencers as potential collaboration partners within the vaccination network. The authors suggest that 'health communication professionals and social influencers can collaborate effectively to create health interventions that are tailored to the preferences, perceptions and cultures of these online communities'. ${ }^{16}$ Whether these concepts can be used to help to tailor social media campaigns to combat medical misinformation requires further research.

\section{GREATER PUBLIC ENGAGEMENT}

Traditional public health engagement campaigns, led by expert health organisations, may be needed to address specific cases of misinformation. It is also possible that public health organisations could benefit from appointing figureheads from medical specialities (for example, 
an experienced and trusted cardiologist) whose specific roles could be to 'advise the general public regarding treatments and to help to dispel misinformation regarding their specialty'. ${ }^{11}$ These public engagement officers could augment the public health-orientated roles performed by public health leaders, such as the Surgeon General in the USA and the Chief Medical Officer in the UK.

It may also be useful to consider novel ways to directly engage with the public to facilitate open dialogue regarding controversial health topics. An interesting example is the 'Ask Me Anything' (AMA) initiative on the social media platform Reddit. ${ }^{17}$ In AMA sessions, experts are invited to answer questions from Reddit users, to encourage a two-way dialogue between experts and the public regarding specific topics. Hara et $a l^{17}$ found that science-themed AMA sessions were perceived positively by expert hosts and suggest that this communication format may be a promising way to actively engage the public in scientific discussion. Whether this approach can be applied to topics of healthcare debate and medical misinformation warrants further study.

\section{DEVELOPING A CULTURE OF FACT- CHECKING}

A key goal should be to foster a culture of fact-checking within the general public. Evidence suggests that people with lower health literacy may be more likely to trust sources of health information on social media and blogs. ${ }^{18}$ By helping laypeople to develop greater scientific and critical health literacy skills, we may empower them to question the validity of health information shared on social media. However, there is limited robust evidence supporting the use of specific interventions to increase online health literacy and improve individuals ability to evaluate claims about the effects of health interventions. ${ }^{19-21}$ Communitybased interventions, including collaborative learning and increased social support, may help to improve older adults critical health literacy. ${ }^{19}$ Web-based educational interventions may improve knowledge and skills in the short-term; however, the long-term effects of these interventions are uncertain. ${ }^{20}$ Further research is urgently needed to identify optimal strategies to increase scientific and health literacy in specific groups; it is likely that interventions will need to be carefully tailored to benefit difficult-to-reach populations.

\section{DOCTORS AS ADVOCATES}

Doctors are well-placed to help to counter medical misinformation online, yet have historically been cautioned against engaging with social media in a professional capacity, due to the risks associated with the blurring of personal and professional boundaries. ${ }^{22}$ However, the threat from medical misinformation is such that it demands a proactive, collaborative response from key stakeholders, including doctors. Doctors may be able to help to mitigate the medical misinformation mess by curating and disseminating evidencebased content to the general public via social media platforms, such as Twitter and Facebook. However, it is important to consider how well a tweet, containing only 280 characters, summarises findings from a complex study; it may be difficult to discuss the nuances and limitations of research using this limited word count. It is, therefore, important that doctors receive appropriate training regarding effective use of social media for health promotion.

In addition, doctors should use patient encounters to communicate scientific consensus and signpost to reliable, evidence-based online resources while cautioning against the reliability of health information shared on social media. General practitioners could even provide leaflets or checklists containing basic tips regarding how to identify red flags for misinformation online. At the very least, an individual doctor or scientist can become a 'nerd of trust' within their own social network. ${ }^{23}$ Whether we like it or not, patients are using the Internet as a medical resource and are obtaining health information via social media. We should use our expertise to create and endorse more reliable, accessible Internet-based patient resources while encouraging and participating in the sharing of factchecked medical information on social media.

\section{SUMMARY}

The sheer volume of medical information online can make it difficult to retrieve reliable, evidence-based information and distinguish medical fact from fiction. The multifaceted nature of medical misinformation means that we cannot expect any single intervention to solve the problem alone. Further research is urgently needed to build our understanding of how medical misinformation spreads via social media and to identify the most effective strategies to address this important public health problem.
Contributors SPT is the sole author.

Funding The author has not declared a specific grant for this research from any funding agency in the public, commercial or not-for-profit sectors.

Competing interests None declared.

Patient consent for publication Not required.

Provenance and peer review Not commissioned; externally peer reviewed.

(C) Author(s) (or their employer(s)) 2020. No commercial re-use. See rights and permissions. Published by BMJ.

$$
\text { Check for updates }
$$

To cite Trethewey SP. Postgrad Med J 2020;96:4-6.

Received 3 October 2019

Accepted 3 November 2019

Published Online First 15 November 2019

Postgrad Med J 2020;96:4-6.

doi:10.1136/postgradmedj-2019-137201

\section{ORCID iD}

Samuel P Trethewey http://orcid.org/0000-0003-01502283

\section{REFERENCES}

1 Larson HJ. The biggest pandemic risk? viral misinformation. Nature 2018;562:309.

2 Madara JL. American Medical association. AMA urges Tech giants to combat vaccine misinformation. Available: https://www.ama-assn.org/press-center/ press-releases/ama-urges-tech-giants-combat-vaccinemisinformation[Accessed 29 Jul 2019].

3 Hill JA, Agewall S, Baranchuk A, et al. Medical misinformation: Vet the message! Circulation 2019:139:571-2.

4 Chou W-YS, Oh A, Klein WMP. Addressing healthrelated misinformation on social media. JAMA 2018:320:2417-8.

5 Sumner P, Vivian-Griffiths S, Boivin J, et al. Exaggerations and caveats in press releases and health-related science news. PLoS One 2016:11:e0168217.

6 Yavchitz A, Boutron I, Bafeta A, et al. Misrepresentation of randomized controlled trials in press releases and news coverage: a cohort study. PLoS Med 2012:9:e1001308.

7 Adams RC, Challenger A, Bratton L, et al. Claims of causality in health news: a randomised trial. BMC Med 2019;17:91.

8 The Academy of Medical Sciences. Enhancing the use of scientific evidence to judge the potential benefits and harms of medicines, 2017. Available: https:// acmedsci.ac.uk/file-download/44970096

9 Ryan G, Sfar-Gandoura H. Disseminating research information through Facebook and Twitter (drift): presenting an evidence-based framework. Nurse Res 2018:26:41-9.

10 Armstrong PW, Naylor CD. Counteracting health misinformation: a role for medical journals? JAMA 2019:321:1863-4.

11 Trethewey SP. Medical misinformation on social media: cognitive bias, Pseudo-Peer review, and the good intentions hypothesis. Circulation 2019;140:1131-3.

12 Vraga EK, Bode L. Using expert sources to correct health misinformation in social media. Sci Commun 2017:39:621-45

13 Endsley MR. Combating information attacks in the age of the Internet: new challenges for cognitive engineering. Hum Factors 2018;60:1081-94.

14 Ecker UKH, O'Reilly Z, Reid JS, et al. The effectiveness of short-format refutational fact-checks. Br J Psychol 2019;95.

15 Burke-Garcia A, Kreps GL, Wright KB. Perceptions about disseminating health information among 
Mommy Bloggers: quantitative study. JMIR Res Protoc 2018;7:e116.

16 Lutkenhaus RO, Jansz J, Bouman MPA. Tailoring in the digital era: stimulating dialogues on health topics in collaboration with social media influencers. Digital Health 2019;5:205520761882152-11.

17 Hara N, Abbazio J, Perkins K. An emerging form of public engagement with science: ask me anything (AMA) sessions on Reddit r/science. PLoS One 2019;14:e0216789.
18 Chen X, Hay JL, Waters EA, et al. Health literacy and use and trust in health information. J Health Commun 2018;23:724-34.

19 de Wit L, Fenenga C, Giammarchi C, et al. CommunityBased initiatives improving critical health literacy: a systematic review and meta-synthesis of qualitative evidence. BMC Public Health 2018;18:40.10.1186/ s12889-017-4570-7

20 Cusack L, Del Mar CB, Chalmers I, et al. Educational interventions to improve people's understanding of key concepts in assessing the effects of health interventions: a systematic review. Syst Rev 2018;7:68.

21 Car J, Lang B, Colledge A, et al. Interventions for enhancing consumers' online health literacy. Cochrane Database Syst Rev 2011;74.

22 Cork N, Grant P. Blurred lines: the general medical Council guidance on doctors and social media. Clin Med 2016;16:219-22.

23 McClain CR. Practices and promises of Facebook for science outreach: Becoming a "Nerd of Trust". PLoS Biol 2017;15:e2002020. 